\title{
A FIXED POINT THEOREM FOR HYPERSPACES OF $\lambda$ CONNECTED CONTINUA
}

\author{
CHARLES L. HAGOPIAN
}

ABSTRACT. Suppose that the hyperspace of compact connected subsets $\mathcal{C}(X)$ of a $\lambda$ connected continuum $X$ can be $\epsilon$-mapped (for each $\epsilon>0$ ) into the plane. We prove that $X$ is either arc-like or circle-like. It follows from this theorem and results of J. T. Rogers, Jr. and J. Segal that $\mathcal{C}(X)$ has the fixed point property.

We call a nondegenerate metric space that is compact and connected a continuum. The hyperspace $\mathcal{C}(X)$ of a continuum $X$ is the space of compact connected subsets of $X$ with the Hausdorff metric $\rho$ (i,e, $\rho(A, B)=$ g.l.b. $\{\epsilon \mid$ $A \subset N_{\epsilon}(B)$ and $B \subset N_{\epsilon}(A)$, where $N_{\epsilon}(A)$ is the $\epsilon$-neighborhood of $\left.\left.A\right\}\right)$ [6].

A map is a continuous single-valued function. A continuum $X$ is said to have the fixed point property if for each map $f$ of $X$ into itself, there is a point $x$ of $X$ such that $f(x)=x$. A map $f$ of a continuum $X$ is called an $\epsilon-m a p$ if for each point $y$ of $f[X]$, the diameter of $f^{-1}(y)$ is less than $\epsilon_{\text {。 }}$

$A$ continuum $X$ is arc-like if for each positive number $\epsilon$, there is an $\epsilon$ map of $X$ onto an arc. Circle-like and disk-like continua are defined in the same manner.

A continuum is decomposable if it is the union of two proper subcontinua. A continuum is hereditarily decomposable if all of its subcontinua are decomposable. If every pair of points in a continuum $X$ lies in a hereditarily decomposable subcontinuum of $X$, then $X$ is said to be $\lambda$ connected.

A continuum $T$ is called a triod if it contains a subcontinuum $Z$ such that $T-Z$ is the union of three nonempty disjoint open sets. When a continuum does not contain a triod, it is said to be atriodic.

A continuum is unicoherent provided that if it is the union of two subcontinua $E$ and $F$, then $E \cap F$ is connected.

Throughout this paper $E^{2}$ is the Euclidean plane and $\delta$ is the standard Euclidean metric on $E^{2}$. The boundary and the interior of a given set $Z$ are denoted by $B d Z$ and Int $Z$ respectively.

Received by the editors September 2, 1974.

AMS (MOS) subject classifications (1970). Primary 54B20, 54C10, 54F20, 54F60, 54H25; Secondary 54C05, 54F 25, 54F55.

Key words and phrases. Hyperspaces, chainable continua, arc-like continua, circle-like continua, fixed point property, lambda connectivity, hereditarily decome posable continua, disk-like continua, triod, snake-like continua, unicoherence, $\epsilon-$ map into the plane, antipodal points, Borsuk-Ulam theorem. 
We define the following subsets of $E^{2}$.

$D_{1}=\{(x, y) \mid 0 \leq x \leq 6$ and $0 \leq y \leq 6\}, \quad D_{2}=\{(x, y) \mid 0 \leq x \leq 5$ and $0 \leq y \leq 6\}$, $D_{3}=\{(x, y) \mid 3 \leq x \leq 4$ and $2 \leq y \leq 4\}, \quad D_{4}=\{(x, y) \mid 3 \leq x \leq 6$ and $0 \leq y \leq 6\}$, $B=\{(x, y) \mid \delta((x, y),(1,2))<1$ or $\delta((x, y),(1,4))<1\}$, $D=D_{1}-B$, and $D^{\prime}=D_{2}-\{(x, y) \mid \delta((x, y),(0,3))<1 / 3\}$.

Lemma. There exists a positive number $\epsilon$ such that if $f$ is an $\epsilon-m a p$ of $D$ into $E^{2}$, then $f\left[\mathrm{Bd} D^{\prime}\right]$ separates $f((1,3))$ from $f((6,3))$ in $E^{2}$.

Proof. Let $k$ be a map of $D^{\prime}$ onto a 2-sphere $S^{2}$ such that (1) $k$ [Bd $\left.D^{\prime}\right]$ is a point of $S^{2}$, (2) $k$ restricted to Int $D^{\prime}$ is a homeomorphism, and (3) if $p$ and $p^{\prime}$ are antipodal points of $S^{2}$, then $k\left[D_{3}\right] \cap\left\{p, p^{\prime}\right\} \neq \varnothing$. Define $\epsilon$ to be a positive number less than $1 / 2$ such that for each pair $p, p^{\prime}$ of antipodal points of $S^{2}, \delta\left(k^{-1}(p), k^{-1}\left(p^{\prime}\right)\right)>\epsilon$.

Let $f$ be an $\epsilon$-map of $D$ into $E^{2}$. Since $f[\mathrm{Bd} B]$ and $f\left[D_{4}\right]$ are disjoint continua, there is a map $f^{*}$ of $D_{1}$ into $E^{2}$ that is an extension of $f$ such that $f^{*}[B] \cap f\left[D_{4}\right]=\varnothing$.

The complementary domain $G$ of $f\left[B d D^{\prime}\right]$ that contains $f((1,3))$ in $E^{2}$ is a subset of $f^{*}\left[D^{\prime}\right]$. To see this, assume the contrary. Let $w$ be a point of $G-f^{*}\left[D^{\prime}\right]$. Let $d$ be the projection map of $E^{2}$ onto the quotient space $E^{2} / R$, where $R$ relates distinct points $z$ and $z^{\prime}$ of $E^{2}$ if and only if $\left\{z, z^{\prime}\right\}$ $C E^{2}-G$. Since $E^{2} / R$ is either a 2 -sphere or a plane, we can assume that $E^{2} / R-\{w\}$ is lying in $E^{2}$. Note that $h=d f^{*} k^{-1}$ is a map of $S^{2}$ into $E^{2}$. Also note that since $f\left[\mathrm{Bd} D^{\prime}\right]$ does not meet the image under $f$ of the line segment in $E^{2}$ from $(1,3)$ to $(3,3)$, the domain $G$ contains $f\left[D_{3}\right]$. According to the Borsuk-Ulam theorem, there exist antipodal points $p$ and $p^{\prime}$ of $S^{2}$ such that $h(p)=h\left(p^{\prime}\right)$. But since $f$ is an $\epsilon$-map and $f^{*}[B] \cap f\left[D_{3}\right]=\varnothing$, this is a contradiction.

Since $f((6,3))$ does not belong to $f^{*}\left[D^{\prime}\right]$, it follows that $f((6,3))$ is not in $G$. Hence $f\left[\mathrm{Bd} D^{\prime}\right]$ separates $f((1,3))$ from $f((6,3))$ in $E^{2}$.

Theorem 1. Suppose that for each $\epsilon>0$ the hyperspace $\mathcal{C}(X)$ of a continuum $X$ can be $\epsilon$-mapped into $E^{2}$. Then $X$ is atriodic and every proper subcontinuum of $X$ is unicoherent.

Proof. Assume that $X$ contains a triod. Then $\mathcal{C}(X)$ contains a 3-cell $B^{3}$ [9, Theorem 1]. It follows from the Borsuk-Ulam theorem that for some $\epsilon>0, \mathrm{Bd} B^{3}$ cannot be $\epsilon$-mapped into $E^{2}$, which contradicts our hypothesis. Hence $X$ is atriodic.

Suppose that a proper subcontinuum $Y$ of $X$ is not unicoherent. Then there exist continua $E_{1}, E_{2}$ and disjoint nonempty closed sets $A_{1}, A_{2}$ such 
that $E_{1} \cup E_{2}=Y$ and $E_{1} \cap E_{2}=A_{1} \cup A_{2}$. Since $X$ is atriodic, $A_{1}$ and $A_{2}$ are components of $A_{1} \cup A_{2}$ [7, Theorem 50, p. 18]. We can assume without loss of generality that $E_{1}$ and $E_{2}$ are both irreducible with respect to intersecting $A_{1}$ and $A_{2}$.

For $i=1,2$ and $j=1,2$, let $\mathbb{G}_{i j}$ be a monotone increasing collection of compact connected subsets of $E_{j}$ that forms an arc in $\mathcal{C}(X)$ from $A_{i}$ to $E_{j}$. Note that $\bigcup_{i, j} \mathbb{G}_{i j}$ is a simple closed curve in $\mathcal{C}(X)$.

For $i=1$ and 2 , let $h_{i}$ be the function of $\mathbb{G}_{i 1} \times \mathbb{G}_{i 2}$ into $\mathcal{C}(X)$ that assigns to each point $\left(X_{1}, X_{2}\right)$ of $\mathbb{G}_{i 1} \times \mathbb{G}_{i 2}$ the compact connected set $X_{1}$ $\cup X_{2}$. Each $h_{i}$ is a homeomorphism. The intersection of the disks $h_{1}\left[\mathbb{Q}_{11} \times \mathbb{Q}_{12}\right]$ and $h_{2}\left[\mathbb{Q}_{21} \times \mathbb{Q}_{22}\right]$ is $\left\{E_{1}, E_{2}, Y\right\}$. In fact, there is a homeomorphism $g$ of $h_{1}\left[\mathbb{G}_{11} \times \mathbb{G}_{12}\right] \cup h_{2}\left[\mathscr{G}_{21} \times \mathbb{G}_{22}\right]$ onto $D$ (the square with two holes in $E^{2}$ defined above) such that $g\left(A_{1}\right)=(0,3), g\left(A_{2}\right)=(6,3), g\left(E_{1}\right)=$ $(0,2), g\left(E_{2}\right)=(0,4)$, and $g(Y)=(1,3)$.

According to our Lemma, there is a positive number $\epsilon_{1}$ such that if $f$ is an $\epsilon_{1}$-map of $\mathcal{C}(X)$ into $E^{2}$, then $f\left[g^{-1}\left[\mathrm{Bd} D^{\prime}\right]\right]$ separates $f(Y)$ from $f\left(A_{2}\right)$ in $E^{2}$.

Let 9 be a monotone increasing collection of subcontinua of $X$ that forms an arc from $Y$ to $X$ in $\mathcal{C}(X)$. Let $u$ be a point of $A_{2}$. If $\{u\}=A_{2}$, define $\mathcal{I}=\left\{A_{2}\right\}$; otherwise, let $\mathcal{I}$ be a monotone increasing collection of compact connected sets in $A_{2}$ that forms an arc in $\mathcal{C}(X)$ from $\{u\}$ to $A_{2}$. Define $v$ to be a point of $X-Y$ and let $K$ be a monotone increasing collection of compact connected sets in $X$ that forms an arc from $\{v\}$ to $X$ in $\mathcal{C}(X)$. Define $\mathcal{S}$ to be the continuum in $\mathcal{C}(X)$ whose points are the singletons of $X$. Note that $\mathbb{M}=\mathfrak{g} \cup \mathfrak{I} \cup \mathcal{K} \cup \mathcal{S}$ is a subcontinuum of $\mathcal{C}(X)$ that contains the set $\left\{Y, A_{2}\right\}$ and misses $g^{-1}\left[\mathrm{Bd} D^{\prime}\right]$.

Define $\epsilon$ to be the minimum of $\epsilon_{1}$ and the distance from $\mathbb{M}$ to $g^{-1}\left[B d D^{\prime}\right]$ in $\mathcal{C}(X)$. Let $f$ be an $\epsilon$-map of $\mathcal{C}(X)$ into $E^{2}$. It follows that the continuum $f[\mathbb{N}]$ contains $\left\{f(Y), f\left(A_{2}\right)\right\}$ and does not intersect $f\left[g^{-1}\left[\mathrm{Bd} D^{\prime}\right]\right]$. But since $f\left[g^{-1}\left[\mathrm{Bd} D^{\prime}\right]\right]$ separates $f(Y)$ from $f\left(A_{2}\right)$ in $E^{2}$, this is a contradiction. Hence every proper subcontinuum of $X$ is unicoherent.

Theorem 2. Suppose that $X$ is a $\lambda$ connected continuum and that for each $\epsilon>0, \mathcal{C}(X)$ can be $\epsilon$-mapped into $E^{2}$. Then $X$ is either arc-like or circle-like.

Proof. $X$ is atriodic and every proper subcontinuum of $X$ is unicoherent (Theorem 1). If $X$ is unicoherent, then $X$ is hereditarily decomposable [3, Theorem 2] and therefore arc-like [1, Theorem 11]. If $X$ is not unicoherent, then $X$ is circle-like [4, Theorem 2].

Theorem 3. If $X$ satisfies the hypothesis of Theorem 2, then $\mathcal{C}(X)$ is disk-like and has the fixed point property. 
Proof. If a continuum $Y$ is an arc or a circle, then $\mathcal{C}(Y)$ is a disk. Therefore, since $X$ is arc-like or circle-like, $\mathcal{C}(X)$ is disk-like [12]. Segal [11] proved that for each arc-like continuum $Y$, the hyperspace $\mathcal{C}(Y)$ has the fixed point property. Rogers [10] showed that $\mathcal{C}(Y)$ has the fixed point property when $Y$ is a circle-like continuum.

Comments. It follows from results in [4] and [5] that true statements are obtained when the phrase "the cone over $X$ " is substituted for " $C(X)$ ", in Theorems 2 and 3. In [3] we proved that $\lambda$ connected continua $X$ and $Y$ are arc-like if and only if $X \times Y$ is disk-like. Hence if $X$ and $Y$ are $\lambda$ connected continua and $X \times Y$ is disk-like, $X \times Y$ has the fixed point property [2]. We do not have an example of a disk-like continuum that does not have the fixed point property.

$\mathcal{C}(X)$ is embeddable in $E^{2}$ if and only if $X$ is an arc or a simple closed curve [8, Theorem 2.3]. For $\epsilon$-mappings we have the following analogue.

Theorem 4. Suppose that $X$ is a $\lambda$ connected continuum. Then $\mathcal{C}(X)$ can be $\epsilon$-mapped (for each $\epsilon>0$ ) into $E^{2}$ if and only if $X$ is arc-like or circle-like.

Question. Can Theorem 4 be extended to include all continua?

\section{REFERENCES}

1. R. H. Bing, Snake-like continua, Duke. Math. J. 18(1951), 653-663. MR 13, 265.

2. E. Dyer, A fixed point theorem, Proc. Amer. Math. Soc. 7 (1965), 662-672. MR 17, 1232.

3. C. L. Hagopian, Disk-like products of $\lambda$ connected continua. I, Proc. Amer. Math. Soc. 51 (1975), 448-452.

4. - , Disk-like products of $\lambda$ connected continua. II, Proc. Amer. Math. Soc. 51 (1975), 448-452.

5. W. Holsztyński, Universal mappings and fixed point theorems, Bull, Acad. Polon. Sci. Sér. Sci. Math. Astronom. Phys. 15 (1967), 433-438. MR 36 \#4545.

6. J. L. Kelley, Hyperspaces of a continuum, Trans. Amer. Math. Soc. 52 (1042), 22-36. MR 3, 315 .

7. R. L. Moore, Foundations of point set theory, rev. ed., Aner. Math. Soc. Colloq. Publ., vol. 13, Amer. Math. Soc., Providence, R. I., 1962.

8. S. B. Nadler, J r., Some problems concerning hyperspaces, Topology Conf. (Virginia Polytechnic Inst. and State Univ., 1973), Lecture Notes in Math., vol 375, Springer-Verlag, New York, 1974, pp. 190-197.

9. J. T. Rogers, Jr., Dimensions of hyperspaces, Bull. Polon. Acad. Sci. 20 (1972), 177-179.

10. - Hyperspaces of arc-like and circle-like continua, Topology Conf. (Virginia Polytechnic Inst. and State Univ., 1973), Lecture Notes in Math., vol. 375, Springer-Verlag, New York, 1974, pp. 231-235.

11. J. Segal, $A$ fixed point theorem for the hyperspace of a snake-like continuum, Fund. Math. 50 (1961/62), 237-248. MR $25 \# 2582$.

12. - Hyperspaces of the inverse limit space, Proc. Amer. Math. Soc. 10 (1959), 706-709. MR $21 \# 7492$.

DEPARTMENT OF MATHEMATICS, CALIFORNIA STATE UNIVERSITY, SACRAMENTO, CALIFORNIA 95819 